\title{
Non-linear label-free imaging through a multimode graded index optical fibre
}

\author{
Angel Cifuentes ${ }^{1, *}$, Johanna Trägårdh ${ }^{l}$, Tomáš Pikálek $^{l}$, Mojmir Šerýl, Denis Akimov ${ }^{2}$, Tobias Meyer $^{2,3}$, Jürgen Popp ${ }^{2,3}$, \\ Rodrigo Amezcua-Correa ${ }^{4}$ and Tomáš Čižmár ${ }^{1,2,5}$ \\ ${ }^{1}$ Institute of Scientific Instruments of the CAS, v. v. i., Královopolská 147, 61264 Brno, Czech Republic \\ ${ }^{2}$ Leibniz Institute of Photonic Technology, Albert-Einstein-Straße 9, 07745 Jena, Germany \\ ${ }^{3}$ Institute of Physical Chemistry, Friedrich Schiller University of Jena and Abbe Center of Photonics, Helmholtzweg 4, 07743 Jena, \\ Germany \\ ${ }^{4}$ CREOL, The College of Optics and Photonics, University of Central Florida, 4304 Scorpius St., 32816-2700 Orlando, Florida, United \\ States of America \\ ${ }^{5}$ Institute of Applied Optics, Friedrich Schiller University Jena, Fröbelstieg 1, 07743 Jena, Germany
}

\begin{abstract}
In recent years, great advances have been made in developing minimal footprint micro-endoscopes using multimode optical fibres (MMF) [1]. By employing wavefront shaping methods the seemingly random speckle pattern resulting from the guiding of coherent light through an MMF can be formed into a diffraction limited spot. This enables the implementation of multiple laser scanning techniques. In this work we show that this approach can be employed to realize label-free non-linear microscopy techniques such as coherent anti-Stokes Raman scattering (CARS) and second harmonic generation (SHG). Backscattered light makes epi-detection possible even though these processes preferably emit light in the direction of beam propagation [2].
\end{abstract}

\section{Introduction}

The potential for MMF to be utilized as micro-endoscopes has come into light in recent years. By measuring the transmission matrix (TM) of a MMF it is possible to obtain a tightly focused spot on the far side of said fibre. The spot can be swept across the facet allowing for imaging techniques to be implemented. Label-free imaging offers the opportunity to observe and analyse samples without the use of exogenous markers, making it particularly alluring for endoscopic applications. Furthermore, graded index (GRIN) MMFs push the potential even further due to their resilience to bending induced distortions [3] and larger bandwidth (allowing the guiding of ultrashort pulses).

In this work we demonstrate the use of GRIN MMFs for imaging using two non-linear label-free techniques, namely, coherent anti-stokes Raman scattering (CARS) and polarization resolved second harmonic generation $(\mathrm{P}-$ SHG).

\section{Methods}

\subsection{Calibration}

We refer to calibration as the procedure used to measure the TM. For this, a set of input and output modes is defined. In our case, the proximal facet of the MMF was placed in the Fourier plane of a SLM and, by displaying the appropriate gratings, a grid of individual input spots, or modes, can be projected onto the proximal facet. Each input mode is addressed one at a time and the resulting speckle pattern at the distal end of the fibre is imaged on to a digital camera. Here, phase shifting interferometry allows for the determination of the complex amplitude of the field. For convenience, the set of output modes is defined by the camera pixels themselves. The process is carried out for each input mode resulting in the TM of the fibre. We may then select a desired output mode and calculate the light pattern required at the fibre proximal end to obtain constructive interference at a single well defined point in the distal end, effectively creating a focus.

The calibration procedure must be repeated for each wavelength used, tuning it without recalibrating is only possible for a small range (tens of nanometres depending on the fibre used). Moreover, an independent calibration must be effectuated for two orthogonal polarization states at the proximal end to improve the efficiency with which light may be focused into a single spot. Additionally, if full control over the polarization state of the resulting spot is required, a set of output polarization orthogonal TMs must be obtained, this can be achieved by rotating the polarization of the reference beam by $90^{\circ}$.

\subsection{Experimental setup}

To perform the calibration and imaging, the setup seen in figure 1 was utilized. A liquid crystal phase-only SLM is used to create the required input patterns on the fibre

\footnotetext{
* Corresponding author: cifuentes@isibrno.cz
} 
proximal facet during calibration and imaging. The orthogonal polarization states of the input light are independently controlled, shaped, and overlapped (both spatially and temporally) before projection onto the fibre facet through a $20 \mathrm{x} / 0.45 \mathrm{NA}$ microscope objective.

On the distal side, a 20x/0.45NA microscope objective and corresponding tube lens image the sample plane onto a digital camera where the speckle pattern is interfered with a reference beam. A delay line is used to obtain the temporal overlap required.

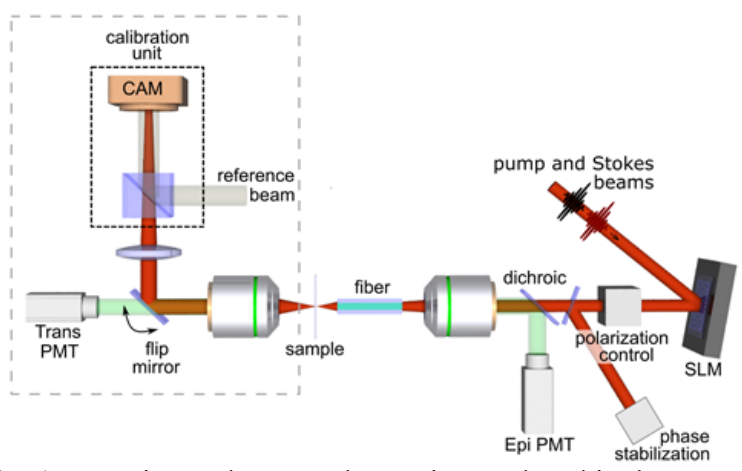

Fig. 1. Experimental setup. The section enclosed in the grey dashed line is only necessary when calibrating or imaging in transmission. Peripheral optical elements are not shown.

\section{Results}

\subsection{CARS}

CARS imaging and its inherent chemical selectivity was demonstrated by imaging $2 \mu \mathrm{m}$ polystyrene beads (PS) and $2.5 \mu \mathrm{m}$ PMMA beads (Figure 2), with a pixel integration time as low as $1 \mathrm{~ms}$ for epi-detection. A 125 $\mu m$ cladding $/ 50 \mu m$ core 0.29 NA GRIN fibre was used. The beams used were 1-2 ps long. The pump beam used was tuneable from $660-675 \mathrm{~nm}$ while the Stokes was fixed at $831 \mathrm{~nm}$. [4]
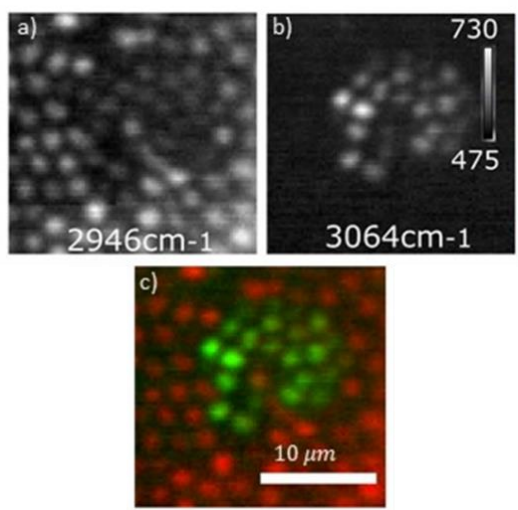

Fig. 2. Epi CARS imaging of polymethyl methacrylate (PMMA) and polystyrene (PS) beads through a GRIN MMF using 1-2 ps laser pulses showing chemical selectivity. a) PMMA beads, Raman shift $=2946 \mathrm{~cm}-1$ b) PS beads, Raman shift $=3064 \mathrm{~cm}-1, \mathrm{c})$ Composite image

\subsection{P-SHG}

In the case of P-SHG a $1040 \mathrm{~nm}, \sim 100$ fs long pulse was used to image a starch grain through a 0.29 NA $62.5 \mu \mathrm{m}$ core $100 \mu \mathrm{m}$ cladding GRIN MMF. The polarization of the exciting spot was varied from $30^{\circ}$ to $180^{\circ}$. An additional image using right-handed circular polarization was also taken. The response of the starch grain to the change in polarization is clearly visible in Fig. 3.

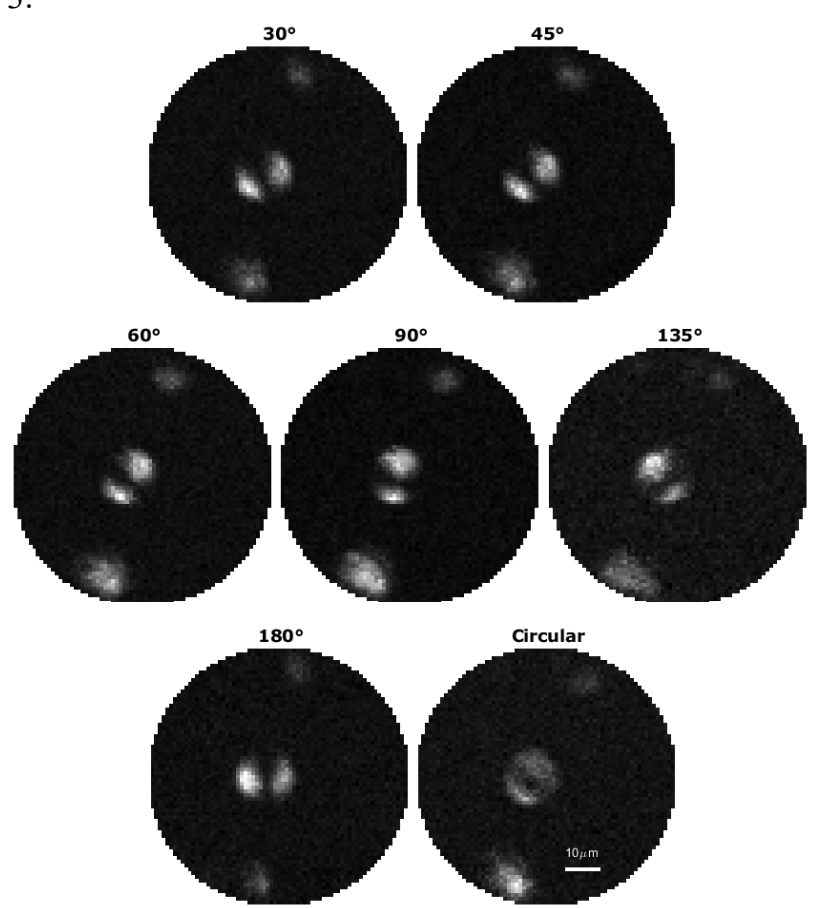

Fig. 3. SHG images of a potato starch grain in epi detection. The polarization angle of the linearly polarized excitation was varied between $30^{\circ}$ and $180^{\circ}$. The resulting image for circular polarization $(\mathrm{RH})$ excitation spot is also shown.

A potential application of $\mathrm{P}-\mathrm{SHG}$ is in determining collagen fibril morphology in tissue. Other biological markers responding to SHG are myosin and microtubules. [5]

\section{Conclusion}

We have shown the capability of GRIN MMF to be used in label-free non-linear applications using the TM based fibre imaging approach. This opens a broad range of possible applications where hard to access samples may be observed in a relatively undisturbed state.

\section{References}

1. S. Turtaev, I. T. Leite, T. Altwegg-Boussac, J. M. Pakan, N. L. Rochefort, T. Čižmár, Light: Science \& Applications 7, 1 (2018).

2. F. Légaré, C. Pfeffer, B. R. Olsen, Biophys. J 93.4, 1312 (2007).

3. D. E. B. Flaes, J. Stopka, S. Turtaev, J. F. De Boer, T. Tyc, T. Čižmár, Physical review letters, 120.23, 233901 (2018).

4. J. Trägårdh, T. Pikálek, M. Šerý, T. Meyer, J. Popp, T. Čižmár, Optics express, 27(21), 3005530066 (2019).

5. R. Cicchi, N. Vogler, D. Kapsokalyvas, B. Dietzek, J. Popp, F.S. Pavone J. Biophotonics 6.2, 129 (2013). 